\title{
Sustained transgene expression despite T lymphocyte responses in a clinical trial of rAAV1-AAT gene therapy
}

\author{
Mark L. Brantlya, Jeffrey D. Chulay', Lili Wangc, Christian Muellerd, Margaret Humphries ${ }^{d}$, L. Terry Spencere, \\ Farshid Rouhania, Thomas J. Conlon ${ }^{f}$, Roberto Calcedoc, Michael R. Betts ${ }^{g}$, Carolyn Spencere, Barry J. Byrnef, \\ James M. Wilsonc, and Terence R. Flotte ${ }^{\mathrm{d}, 1}$
}

aDepartment of Medicine, University of Florida, Gainesville, FL 32611; bApplied Genetic Technologies Corporation, Alachua, FL 32615; 'Department of Pathology and Laboratory Medicine, University of Pennsylvania, Philadelphia, PA 19104; ${ }^{\mathrm{d} D e p a r t m e n t}$ of Pediatrics and Gene Therapy Center, University of Massachusetts, Worcester, MA 01655; eBoston Children's Hospital, Boston, MA 02115; fPowell Gene Therapy Center, University of Florida, Gainesville, FL 32610; 9Department of Microbiology, University of Pennsylvania, Philadelphia, PA 19104

Edited by Inder M. Verma, The Salk Institute, La Jolla, CA, and approved July 7, 2009 (received for review April 28, 2009)

\begin{abstract}
Alpha-1 antitrypsin (AAT) deficiency is well-suited as a target for human gene transfer. We performed a phase 1, open-label, doseescalation clinical trial of a recombinant adeno-associated virus (rAAV) vector expressing normal (M) AAT packaged into serotype 1 AAV capsids delivered by i.m. injection. Nine AAT-deficient subjects were enrolled sequentially in cohorts of 3 each at doses of $6.9 \times 10^{12}$, $2.2 \times 10^{13}$, and $6.0 \times 10^{13}$ vector genome particles per patient. Four subjects receiving AAT protein augmentation discontinued therapy $\mathbf{2 8}$ or $\mathbf{5 6}$ days before vector administration. Vector administration was well tolerated, with only mild local reactions and 1 unrelated serious adverse event (bacterial epididymitis). There were no changes in hematology or clinical chemistry parameters. M-specific AAT was expressed above background in all subjects in cohorts 2 and 3 and was sustained at levels $0.1 \%$ of normal for at least 1 year in the highest dosage level cohort, despite development of neutralizing antibody and IFN- $\gamma$ enzyme-linked immunospot responses to AAV1 capsid at day 14 in all subjects. These findings suggest that immune responses to AAV capsid that develop after i.m. injection of a serotype 1 rAAV vector expressing AAT do not completely eliminate transduced cells in this context.
\end{abstract}

AAV | CTL | immune response | pulmonary | gene therapy

A lpha-1 antitrypsin (AAT) deficiency is among the most common single gene disorders in North America and Northern Europe, with a mutant allele frequency of up to $4 \%(1,2)$. It is inherited as a codominant disorder with variable penetrance. Normal serum AAT levels range from 20 to $53 \mu \mathrm{M}$, and AATdeficient individuals with plasma AAT levels $<11 \mu \mathrm{M}$ are prone to spontaneous development of chronic lung disease, characterized by airway inflammation and panacinar emphysema. More than $95 \%$ of deficient alleles are of one particular proteinase inhibitor (PI) phenotype, known as PI*Z (glu342lys) (3, 4). Heterozygous carriers with $1 \mathrm{PI}^{*} \mathrm{Z}$ allele and 1 wild-type (PI*M) allele may also have an increased risk of lung disease if they are exposed to tobacco smoke or other detrimental environmental factors (5). It is generally accepted that AAT lung disease is caused by a lack of normal function of AAT as the principal plasma antiprotease, and the only currently available treatment is protein augmentation therapy using AAT purified from normal human donor plasma (6). However, protein augmentation therapy is suboptimal because of the need for weekly i.v. infusions, high cost, and limited availability.

On the basis of these considerations, recent attempts at AAT gene therapy have focused on gene augmentation through i.m. administration of recombinant adeno-associated virus (rAAV) vectors, which are capable of long-term transduction of skeletal myofibers in mice, with sustained secretion of plasma proteins, such as AAT, erythropoietin, or factor IX (7-10). A previous phase 1 trial of i.m. rAAV-AAT based on the well-studied AAV serotype 2 showed favorable safety characteristics, but transgene expression was only barely above background transiently in 1 of 12 subjects (11). Because of a marked efficiency advantage for vectors packaged in AAV serotype 1 capsid and injected in murine muscle, our group proceeded with preclinical development of a cross-packaged or pseudotyped rAAV-AAT vector, using the same rAAV-AAT gene cassette as in the previous clinical trial but packaged in a serotype 1 capsid $(10,12,13)$.

Recent data from other clinical programs attempting to use rAAV vectors to augment plasma proteins have raised the possibility that a fundamental limitation to this approach may be posed by immune responses to the AAV capsid. In a recent trial of rAAV2-factor IX delivered by hepatic artery injection, 1 subject developed a transient rise in transaminases concurrent with a loss of transgene expression, whereas a second subject showed transient expression of factor IX without transaminase elevations. A third subject demonstrated transient elevations in transaminases, which correlated with the appearance of T cells specific for the AAV2 capsid without detectable factor IX (cells were not available from the first 2 subjects to evaluate the time course of $\mathrm{T}$ cell activation) (14). Although it is unclear how this observation might relate to other AAV serotype capsids and other routes of administration, preliminary results of a rAAV1-lipoprotein lipase (LPL) trial raised the possibility that such a mechanism might be generalized to i.m. delivery of different serotypes, based on the demonstration of capsid-specific $\mathrm{T}$ cells concurrent with a transient rise in serum creatine kinase $(\mathrm{CK})$ and a reversal of a decline in serum triglycerides (an indirect measure of LPL expression) in 1 subject (15).

In the present study, 9 AAT-deficient (PI*ZZ) individuals were dosed sequentially in cohorts of 3 , each receiving i.m. doses of $6.9 \times$ $10^{12}, 2.2 \times 10^{13}$, or $6.0 \times 10^{13}$ vector genome particles $(\mathrm{vg})$, and immune responses were monitored by IFN- $\gamma$ enzyme-linked im-

Author contributions: J.D.C., M.H., B.J.B., J.M.W., and T.R.F. designed research; L.W., C.M., M.H., L.T.S., F.R., T.J.C., R.C., M.R.B., C.S., B.J.B., J.M.W., and T.R.F. performed research; M.L.B. and T.R.F. contributed new reagents/analytic tools; M.L.B., J.D.C., L.W., C.M., M.H. M.R.B., B.J.B., J.M.W., and T.R.F. analyzed data; and J.D.C., C.M., J.M.W., and T.R.F. wrote the paper.

Conflict of interest statement: T.R.F. and B.J.B. hold patents related to this research, and any monies received have been donated to the Department of Pediatrics, College of Medicine at the University of Florida. T.R.F. and B.J.B. were also founders of Applied Genetic Technologies Corporation (AGTC), the study sponsor. They do not currently hold a position with the company, and any proceeds from this study are donated to the University of Florida. T.R.F.'s laboratory receives research funding from the Alpha 1 Foundation. M.L.B. receives research funding from, holds an endowed professorship from, and is a consultant for the Alpha 1 Foundation. The University of Florida owns an interest in the study agent and also owns stock in the sponsor of the study, AGTC. The Alpha 1 Foundation is closely affiliated with AlphaNet, which owns an interest in the study agent. J.M.W. is an inventor on patents including those involving AAV1 that have been licensed to various biopharmaceutical companies.

This article is a PNAS Direct Submission.

${ }^{1}$ To whom correspondence should be addressed. E-mail: terry.flotte@umassmed.edu.

This article contains supporting information online at www.pnas.org/cgi/content/full/ 0904514106/DCSupplemental. 
Table 1. Demographic and baseline characteristics of subjects

\begin{tabular}{|c|c|c|c|c|c|c|c|}
\hline Subject & Age (y) & Gender & Weight (kg) & $\begin{array}{c}\text { FEV1 } \\
\text { (\% predicted) }\end{array}$ & $\begin{array}{c}\text { AAT protein } \\
\text { augmentation therapy }\end{array}$ & $\begin{array}{c}\text { Prior rAAV2 } \\
\text { vector dose }(\mathrm{vg})\end{array}$ & $\begin{array}{c}\text { Dose of rAAV1 vector } \\
\text { in present trial (vg) }\end{array}$ \\
\hline 101 & 66 & $\mathrm{~F}$ & 54.6 & 53.6 & No & $6.9 \times 10^{12}$ & $6.9 \times 10^{12}$ \\
\hline 102 & 73 & $\mathrm{~F}$ & 66.5 & 86.7 & Yes & - & $6.9 \times 10^{12}$ \\
\hline 103 & 69 & $M$ & 73.7 & 92.1 & Yes & $6.9 \times 10^{12}$ & $6.9 \times 10^{12}$ \\
\hline 201 & 38 & $\mathrm{M}$ & 83.0 & 40.5 & Yes & - & $2.2 \times 10^{13}$ \\
\hline 202 & 54 & $\mathrm{M}$ & 89.0 & 93.0 & No & $6.9 \times 10^{13}$ & $2.2 \times 10^{13}$ \\
\hline 203 & 59 & $M$ & 72.7 & 50.8 & No & $2.1 \times 10^{13}$ & $2.2 \times 10^{13}$ \\
\hline 301 & 47 & $\mathrm{~F}$ & 69.3 & 58.0 & No & - & $6.0 \times 10^{13}$ \\
\hline 302 & 35 & $\mathrm{~F}$ & 134.6 & 97.6 & No & - & $6.0 \times 10^{13}$ \\
\hline 303 & 61 & $M$ & 72.6 & 54.9 & Yes & - & $6.0 \times 10^{13}$ \\
\hline
\end{tabular}

FEV1, forced expiratory volume in $1 \mathrm{~s}$; vg, vector genome particles of rAAV2-AAT; F, female; M, male.

munospot (ELISPOT), lymphocyte proliferation, and serum antibody responses to AAV1 capsid and the AAT transgene. ELISPOT assays were performed prospectively in the last 5 subjects in the trial and retrospectively in others. All other assays were performed prospectively in all patients, as were general safety assessments, serum CK measurements, and assays for transgene expression. These studies have enabled a prospective analysis of the relationship between immune responses to capsid and sustained transgene expression, and have supported the concept that immune responses to the rAAV1-AAT vector do not completely eliminate transduced muscle cells in this context.

\section{Results}

Characteristics of the Study Population. All study subjects were enrolled at the University of Florida under a protocol approved by the Western Institutional Review Board and reviewed by an executive group of the University of Florida Institutional Review Board and the gene therapy Data and Safety Monitoring Board of the National Heart, Lung, and Blood Institute. An Investigational New Drug Application sponsored by Applied Genetic Technologies Corporation was filed with the U.S. Food and Drug Administration. Informed consent was obtained from each subject, and a decision monitoring protocol was used to ensure that subjects understood the risks of the protocol and the anticipated lack of clinical benefit from their participation.

The study population consisted of 9 subjects ( 5 male, 4 female) who had been diagnosed as homozygous for PI* $\mathrm{Z}$ with a serum AAT level $<11 \mu \mathrm{M}$. The demographic and baseline characteristics of the subjects are presented in Table 1. Four subjects had received a serotype 2 AAV-AAT vector in a prior gene transfer study (11). Four subjects were receiving weekly AAT protein augmentation therapy, which was discontinued 28 days (subjects 102 and 103) or 56 days (subjects 201 and 303) before administration of rAAV1AAT and resumed after the day- 90 visit. Subjects were enrolled sequentially into 1 of 3 dosage level cohorts of $6.9 \times 10^{12}, 2.2 \times 10^{13}$, or $6.0 \times 10^{13} \mathrm{vg}$ administered by i.m. injection in a total volume of $9.9 \mathrm{~mL}$.

General Safety and Biodistribution. Each subject underwent extensive clinical and laboratory monitoring at baseline and for 1 year after study agent administration. Minor adverse events were occasionally observed, but the only such events related to the study agent were minimal to mild reactions at the injection site in 7 of 9 subjects (bruising in 6 , swelling or induration in 3 , redness in 2 , and warmth or tenderness in 1 each) that were not considered clinically important. Only 1 serious adverse event was reported, a culture-proven Escherichia coli epididymitis with onset $2 \frac{1}{2}$ weeks after vector administration that was judged unrelated to vector administration. There were no clinically important changes in hematology, serum chemistry, or urinalysis parameters after vector administration.

Biodistribution studies demonstrated vector DNA in the blood in 2 of 3 subjects in each of the 2 lower dose cohorts and 3 of 3 subjects in the highest dose cohort, which was maximal at day 1 and progressively decreased to become negative by day 14 or day 90 in all but 1 subject (supporting information Table S1). No vector DNA was detected in semen.

Time Course of Vector-Mediated Expression of Wild-Type (M) AAT. Vector-mediated expression of AAT was monitored using assays of both total AAT (nephelometry) and wild-type (M) AAT (mAbbased ELISA). Total AAT levels ranged from 3.1 to $6.0 \mu \mathrm{M}$ at day -1 and, except for subject 103 who had a transient increase of Z-type AAT to $11 \mu \mathrm{M}$ concurrent with his bacterial epididymitis, consistent with AAT being an acute-phase reaction protein, did not change appreciably at subsequent visits (mean \pm SD ratio of subsequent to baseline values, $100.8 \% \pm 15.9 \%$ ).

For the 4 subjects who had been receiving AAT protein augmentation therapy, measurement of M-type AAT levels was complicated by the reactivity of the M-specific $\mathrm{mAb}$ to residual infused M-AAT protein therapy. This antibody has more than 500-fold greater affinity for M-type AAT than for Z-type AAT, and background M-specific AAT levels are therefore very low in PI*ZZ individuals (mean $\pm \mathrm{SD}, 10.7 \pm 6.0 \mathrm{nM}$ for $20 \mathrm{PI}^{*} \mathrm{ZZ}$ individuals; upper $99 \%$ confidence interval, $<25 \mathrm{nM}$ ). However, because weekly AAT protein augmentation therapy achieves peak serum levels $>300 \mu \mathrm{M}$ and nadir levels $>11 \mu \mathrm{M}$, the reactivity of the mAb resulted in measured levels of M-specific AAT at day -1 of $235 \mathrm{nM}$ and $358 \mathrm{nM}$ in subjects 102 and 103, who had discontinued protein augmentation therapy 28 days before vector administration, and these levels progressively decreased until day 60 and remained stable at $\approx 13 \mathrm{nM}$ through day 90 . Subjects 201 and 303, who had discontinued protein augmentation therapy 56 days before vector administration, had measured levels of M-specific AAT at day -1 of $33 \mathrm{nM}$ and $18 \mathrm{nM}$, respectively, and these levels decreased to $<10$ $\mathrm{nM}$ at day 14, after which they increased to a maximum of $28 \mathrm{nM}$ on day 90 in subject 201 and $48 \mathrm{nM}$ on day 90 in subject 303 (Fig. 1). Serum M-specific AAT concentrations could not be determined after day 90 in these patients because they had resumed protein augmentation therapy.

Among the 5 subjects who were not receiving AAT protein augmentation therapy, subject 101 received the lowest vector dose and had no appreciable change in M-specific AAT levels. Among those who received the intermediate vector dose, subject 202 had an average pretreatment M-specific AAT level of $10 \mathrm{nM}$ that increased to $18 \mathrm{nM}$ on day 30 and slowly decreased to pretreatment levels by day 90, and subject 203 had an average pretreatment M-specific AAT level of $11 \mathrm{nM}$ that increased to $21 \mathrm{nM}$ on day 60 and then decreased to pretreatment levels by day 180 (Fig. 1). Among those who received the highest vector dose, subject 301 had an average pretreatment M-specific AAT level of $10 \mathrm{nM}$ that increased to 43 $\mathrm{nM}$ on day 90 and remained above $40 \mathrm{nM}$ for 1 year, and subject $302 \mathrm{had}$ an average pretreatment M-specific AAT level of $8 \mathrm{nM}$ that increased to $18 \mathrm{nM}$ on day 45 and then seemed to decrease to 

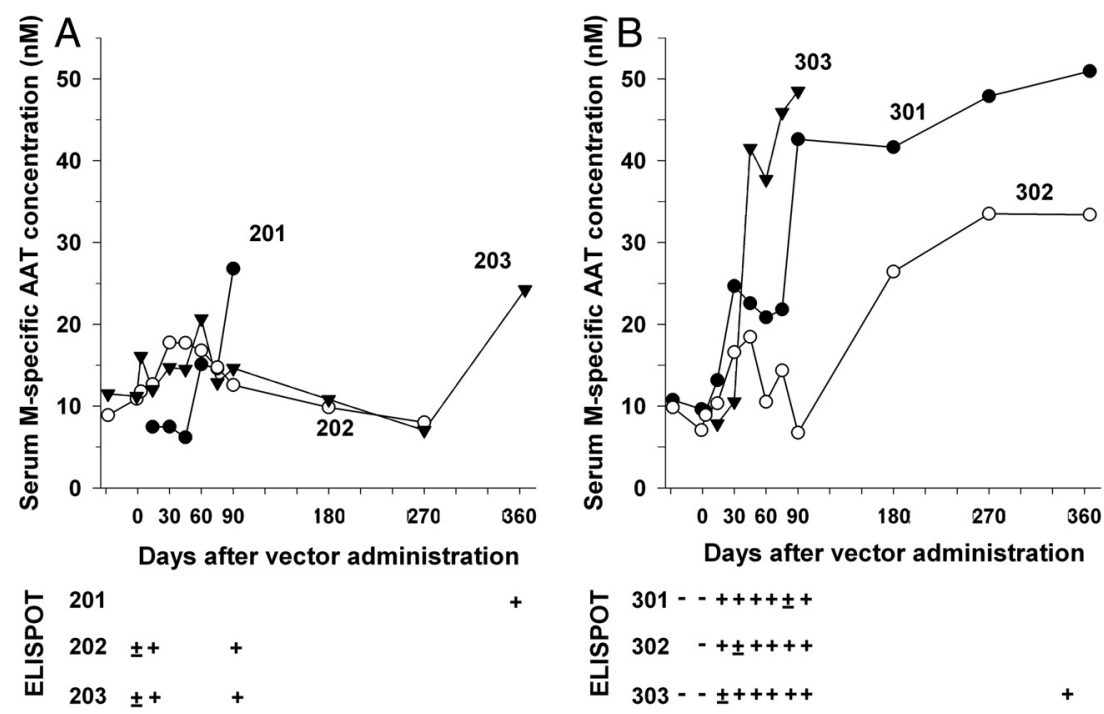

Fig. 1. Time course of vector-mediated AAT expression and ELISPOT responses to AAV1 capsid peptides in $(A)$ cohort 2 and $(B)$ cohort 3. Serum M-specific AAT levels are plotted at the top of each panel and ELISPOT responses are plotted at the bottom of each panel. For subjects 201 and 303 , who had discontinued AAT protein augmentation therapy 56 days before vector administration and resumed protein augmentation soon after day 90, AAT values are not plotted on day -1 , day 3 , or after day 90 but are described in the text. The day-365 sample for subject 202 could not be tested owing to severe hemolysis. ELISPOT responses are characterized as - (negative in both ex vivo and cultured assays), \pm (positive in cultured assay but negative in ex vivo assay), or + (positive in both ex vivo and cultured assays). Samples for ELISPOT analysis were not available beyond day 90 for subjects 202,203 , and 302.

pretreatment levels by day 90 but rose to $26 \mathrm{nM}$ at day 180 and 33 nM at day 270 and day 365 (Fig. 1).

Time Course of Immune Responses to AAT and AAV and CK Levels. Compared with a reference nonhuman primate positive control serum assigned a value of $1 \mathrm{U} / \mathrm{mL}$, anti-AAT antibody levels measured by ELISA were very low $(<1 \mu \mathrm{U} / \mathrm{mL})$ at baseline and remained very low without appreciable change after vector administration (Table S2). All subjects had anti-AAV antibodies detected by ELISA that increased after vector administration (Table S2).

As expected, AAV neutralizing antibody levels at baseline differed between subjects who had or had not previously received a rAAV2 vector (Table S3). Among the 4 subjects who had received the rAAV2 vector, all had substantial neutralizing antibody titers against AAV2 at baseline (range, 160 to 2,560) and lower baseline titers against AAV1 (range, $<20$ to 640 ). Among the 5 subjects who not had received the rAAV2 vector, only 1 (subject 302 ) had a baseline titer (640) against AAV2 and a low but detectable titer (20) against AAV1. After i.m. injection of the rAAV1-AAT vector, all subjects had a clear increase in neutralizing antibody titers against AAV1 and a more variable increase in neutralizing antibody titers against AAV2. There was no relationship between the neutralizing antibody titer and the antibody titer measured by ELISA.

We also measured neutralizing antibody responses to AAV serotypes 7, 8, and rh32.33 (Table S3). Consistent with previously published results (16), antibodies to AAV7 and AAV8 were detected at baseline infrequently (in 2 of 9 subjects, both of whom had received the rAAV2 vector in a previous study) and antibodies to AAVrh32.33 were not detected at baseline. After administration of the rAAV1-CMV enhancer/chicken beta actin promotor-human AAT (rAAV1-CB-hAAT) vector there was a modest increase in antibody titers to AAV7 and AAV8 in 8 of 9 subjects and to AAVrh32.33 in 6 of 9 subjects.

$\mathrm{T}$ cell immune responses to AAV and AAT were evaluated by IFN- $\gamma$ ELISPOT assay and were obtained retrospectively in 3 of the first 4 subjects and prospectively in the last 5 subjects. When tested more than 1 year after vector administration, subjects 101 and 103 had negative responses in an ex vivo ELISPOT assay but positive responses in a cultured ELISPOT assay, and subject 201 had a positive response in both the ex vivo ELISPOT assay and cultured ELISPOT assay (Fig. 1). For the subjects evaluated prospectively, the baseline ex vivo ELISPOT response was negative in all 5, and the cultured ELISPOT response was positive in the 2 subjects (202 and 203) who had previously received the rAAV2-AAT vector and negative in the other 3. All 5 developed positive IFN- $\gamma$ ELISPOT responses by day 14 in the cultured ELISPOT assay (Fig. 1) and by day 14 in 4 and day 30 in the fifth in the ex vivo assay (Fig. 2). All positive responses were directed against at least 2 of the $3 \mathrm{AAV}$ capsid peptide pools, remained positive at day 90 , and were positive at most intervening time points tested. Samples from later time

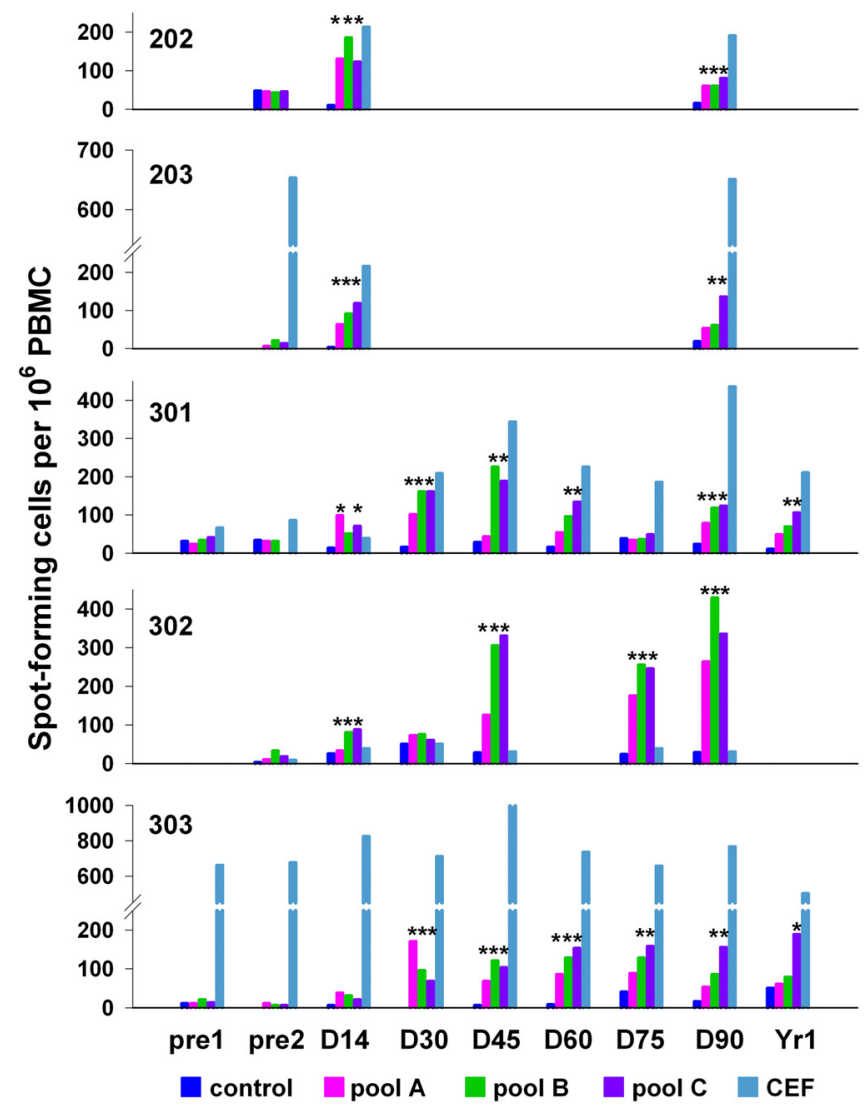

Fig. 2. Time course of IFN- $\gamma$ ELISPOT responses to pools of AAV1 capsid peptides or controls. PBMC were obtained at 1 or 2 times before (pre1 and pre2) and at 14, $30,45,60,75$, and 90 days (D14 through D90, respectively) and 425 or 321 days (Yr1) after vector administration and stimulated with each of 3 pools ( $A, B$, and C) of AAV1 capsid peptides (15-mers overlapping by $10 \mathrm{aa}$ ) or with a positive control peptide pool (CEF). Responses that were significantly increased compared with medium alone (control) are indicated by an asterisk. 
A
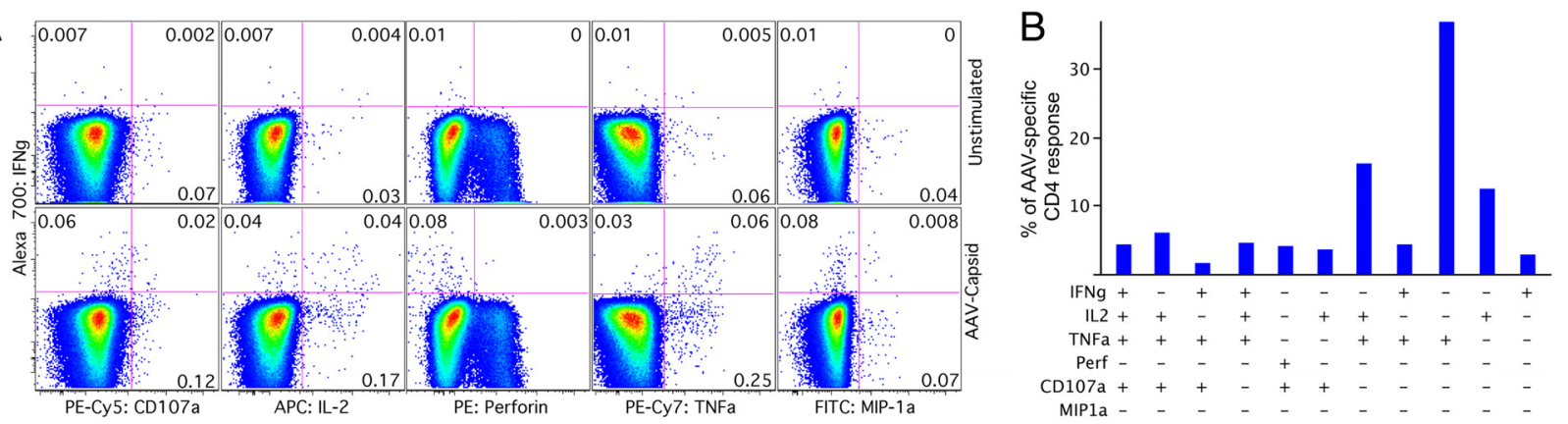

C
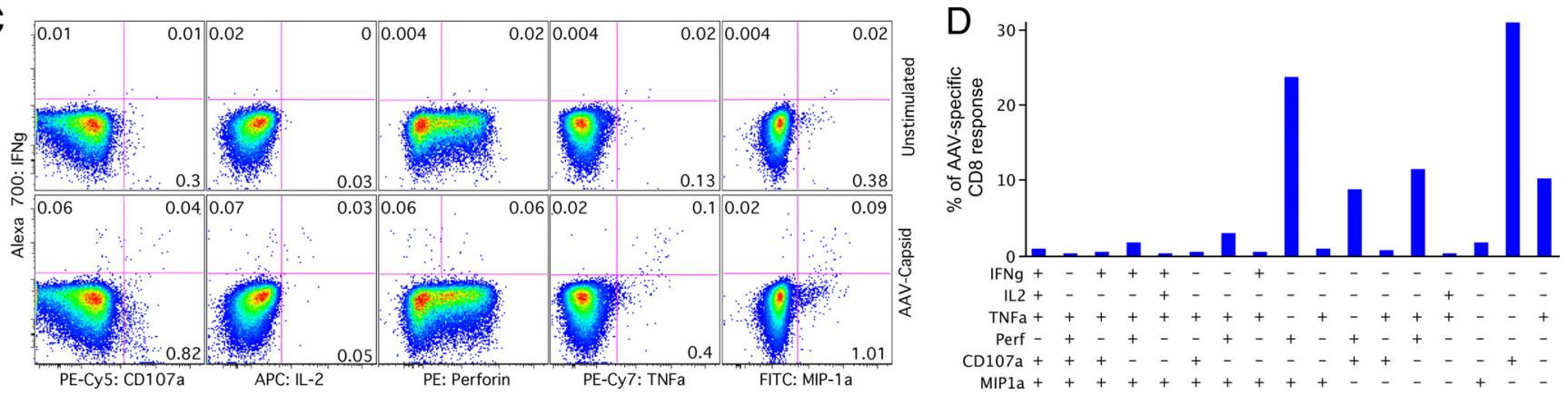

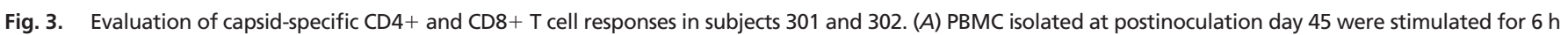

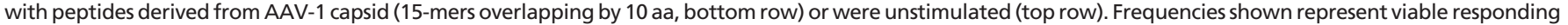

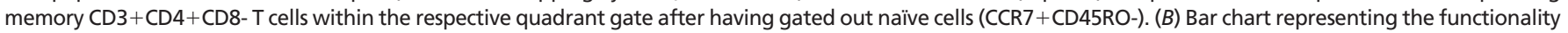

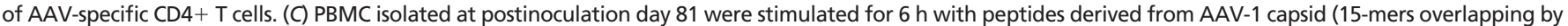

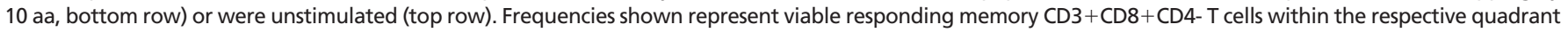

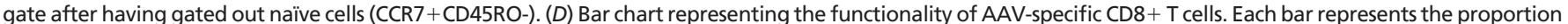

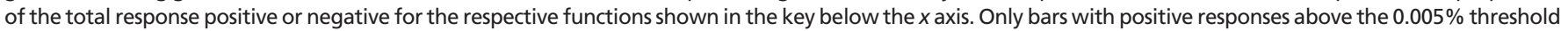
are shown.

points were available for subject 301 (at day 425) and subject 303 (at day 321) and were also positive. The magnitude of the response to any individual pool was generally less than 200 spot-forming cells (SFC) per $10^{6}$ peripheral blood mononuclear cells (PBMC) for 4 subjects but was higher in subject 302 (e.g., the response to pool 3 was $330 \mathrm{SFC} / 10^{6} \mathrm{PBMC}$ at day 45 , and the response to pool 2 was $428 \mathrm{SFC} / 10^{6} \mathrm{PBMC}$ at day 90$)$.

We further investigated by polychromatic flow cytometry the phenotype and functionality of the capsid-specific T cells from 2 subjects. PBMC from day 45 of subject 301 and day 81 of subject 302 were stimulated for $6 \mathrm{~h}$ with the AAV1 peptide library and assessed simultaneously for $6 \mathrm{CD} 8+\mathrm{T}$ cell functions, including degranulation (CD107 $\alpha$ and perforin), production of cytokines (IFN- $\gamma$, TNF- $\alpha$, and IL-2), and chemokine (MIP-1 $\alpha$ ) and cellsurface markers (CD3, CD4, CD8, CD27, CD45RO, CD57, and CCR7) to determine whether the capsid-specific $\mathrm{T}$ cells were CD4+ or CD8+ cells and the functional profile of the responding T cells. Subject 301 had a response to AAV1 capsid mediated by CD $4+$ cells that predominantly expressed TNF- $\alpha$ and IL-2 (Fig. 3 $A$ and $B)$ and expressed markers indicative of central memory phenotype (CCR7+CD27+CD45RO+). Subject 302 had a response to AAV1 capsid mediated by CD $8+\mathrm{T}$ cell that generally seemed effector-like in functionality, with expression of perforin, MIP-1 $\alpha$, CD107a, and TNF- $\gamma$ in various combinations, with relatively little IL-2 and IFN- $\gamma$ production (Fig. $3 C$ and $D$ ). All ex vivo and cultured ELISPOT assays using AAT peptides were negative at all time points tested.

$\mathrm{T}$ cell responses were also evaluated in all subjects using a lymphocyte proliferation assay performed at baseline, day 14, and day 90 . For the AAV antigen, subject 202 had a stimulation index (SI) $<1$ at baseline and day 14 and an SI of 7.0 at day 90 . In subject 301 the SI was 3.3, 0.7, and 2.6 at baseline and days 14 and 90, respectively. All other subjects had negative responses $(\mathrm{SI}<2.5)$ to both AAV and AAT antigens at all time points tested.

Serum CK values were also assessed over time (Fig. 4). Only 5 of the 36 values measured were above the reference range $(>170$ $\mathrm{U} / \mathrm{L})$. Of these, 3 were baseline values, and the other 2 were from 1 of the subjects (102) who had had a high baseline value. None of the values was greater than 1.5 times the upper limit of normal, and none of the subjects for whom an effector $\mathrm{T}$ cell response was demonstrated by day 14 had an elevated CK value after vector administration.

\section{Discussion}

In this first-in-human phase 1 study, we have observed reproducible expression of wild-type M-AAT protein from a crosspackaged rAAV1-AAT vector after i.m. administration that was sustained at subtherapeutic levels for at least 1 year in 2 subjects and at least 90 days in the third subject in the $6.0 \times 10^{13} \mathrm{vg}$ dosage level cohort, despite effector $\mathrm{T}$ cell responses that developed within the first 14 days after vector delivery and persisted 90 days

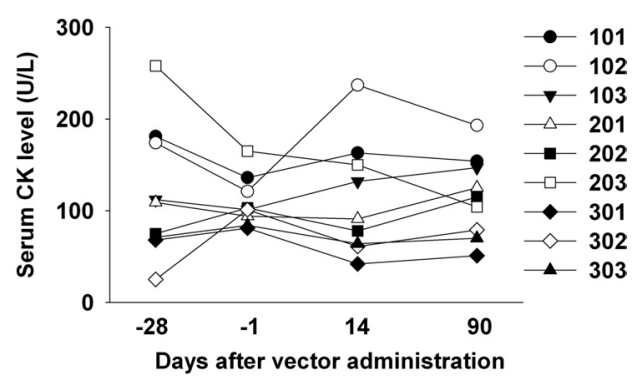

Fig. 4. Serum CK levels before and after vector administration. 
or longer. The sustained nature of the expression of M-AAT, along with the lack of CK elevation in the ELISPOT-positive individuals, leads us to conclude that these responses are not causing complete immune-mediated elimination of vectortransduced myofibers.

Our studies should be considered in the context of the growing body of clinical and preclinical studies evaluating the role of capsid-specific T cells in rAAV gene therapy. The fact that capsidspecific $T$ cells can be generated after in vivo administration of rAAV is well documented in many preclinical studies and in the previously mentioned hemophilia and LPL deficiency human trials. The controversial aspect of the capsid-specific $\mathrm{T}$ cell hypothesis is whether the vector sensitizes transduced cells to become targets for cytotoxic T lymphocyte (CTL)-mediated clearance by virtue of MHC presentation of peptides from the input capsid protein. Our study provides the most direct and extensive test of this hypothesis because we observed sustained transgene expression for at least 1 year in 2 subjects (301 and 302) and at least 90 days in 2 subjects (201 and 303), and no CK elevations, despite the early onset and persistence of $\mathrm{T}$ cell responses to AAV capsid peptides in all subjects in whom these responses were measured. These data clearly demonstrate that transgene expression can persist, despite measured capsid-specific $\mathrm{T}$ cells, without apparent toxicity or attenuation of transgene expression.

A number of hypothetical models might explain this finding. Analysis of $\mathrm{T}$ cell function was limited owing to the availability of cells and the low frequency of antigen-specific T cells. However, 1 of 2 subjects evaluated showed clear evidence of a CD8+ perforin+ CTL response at 81 days after injection, and this subject (302) demonstrated persistently positive transgene expression at 1 year after vector administration. This would suggest that transduced myofibers did not become CTL targets because they did not present sufficient AAV1 capsid peptide on their cell surface. The rAAV vectors used in these studies do not contain AAV capsid genes and therefore cannot express capsid proteins de novo. Thus, it seems quite plausible that transduced myofibers would not display AAV capsid antigens.

The relationship between host and vector in the context of gene therapy is complex and seems to vary considerably according to vector type, serotype, dose, and route of administration, as well as the species being studied. The complexity of these interactions has confounded attempts to predict or model vector behavior in the setting of phase 1 clinical trials. This complexity also comes into play when comparing our data with that in the rAAV2-factor IX clinical trial (14). Key differences between the trials include the serotype of the vector and the route of administration. The rAAV-factor IX study used a serotype 2 vector delivered to the liver by intraportal injection and achieved serum factor IX concentrations up to 0.5 $\mu \mathrm{g} / \mathrm{mL}$ ( $10 \%$ of the normal 4 to $5 \mu \mathrm{g} / \mathrm{mL}$ ). Our trial used a serotype 1 vector delivered to muscle by i.m. injection and achieved serum AAT concentrations up to $2.6 \mu \mathrm{g} / \mathrm{mL}(50 \mathrm{nM})$. It is also possible that the AAT transgene itself, which has anti-inflammatory activity (17), could have exerted an immunomodulatory effect.

Sustained expression of AAT was seen in 1 of 3 subjects at the middle dosage level and all 3 subjects at the highest dosage level tested. The other 2 subjects at these dosage levels had modest increases in AAT expression that were not sustained (although subject 203 had elevated M-specific AAT in a sample at 1 year after treatment). The lack of sustained expression may merely reflect the fact that the expression levels were only modestly above baseline, and even a slight decline would result in an inability to detect continuing low-level expression. The time to peak expression was at least 90 days, which is longer than would have been predicted from nonclinical studies.

The serum neutralizing antibody responses to AAV1 in this trial were consistent and predictable. Some individuals developed high titers of neutralizing antibodies to distinct AAV serotypes such as AAV7, -8, and -32.33, which is unexpected on the basis of animal immunization studies that indicate few shared B cell epitopes between these AAVs $(18,19)$. The diversity in the breadth of serologic responses to intramuscular AAV1 is quite striking, as illustrated by the high-dose group in which 2 subjects (301 and 302) showed a broad response and 1 (303) showed a narrow sero-specific response. It is possible that the endogenous flora of latent/persistent AAVs and resulting immune responses contribute to these unexpected findings. This also suggests that the strategy of using a sequence of vectors with different serologic capsids to overcome the humoral immune response of the first vector may not work in some individuals.

Importantly, none of the subjects developed antibody or $\mathrm{T}$ cell responses to the AAT transgene. This is consistent with clinical experience with AAT augmentation therapy, in which immune responses to AAT have not been observed.

Evaluation of vector biodistribution in this clinical study was limited to collection of blood and semen samples. The detection of vector DNA in blood during the first week after injection, with rapid clearance to low or undetectable levels at day 14 or day 90 , is consistent with results from preclinical studies in mice and rabbits (13). In rabbits, low levels of vector DNA were found in semen during the first week after injection but not at days 21,60 , or 90 , and in the clinical trial no vector DNA was found in semen from any of the men from whom semen samples were available.

The study design originally required subjects receiving AAT augmentation therapy to discontinue that therapy 28 days before vector administration, on the basis of published data indicating that the elimination half-life of infused AAT is 2 to 3 days $(20,21)$. Although this resulted in total AAT levels that were well within the expected range for $\mathrm{PI}^{*} \mathrm{ZZ}$ subjects at day -1 , substantial residual levels of M-type AAT were detected in these subjects, and the "washout" period was extended to 56 days for subjects enrolled in cohorts 2 and 3. However, 2 such subjects had slightly elevated M-type AAT levels at day -1 and day 3 . A recent report suggests that the elimination half-life of infused AAT may be as high as 8 or 9 days (22), and the "washout" period should probably be extended to 90 days in future rAAV-AAT gene transfer studies.

With regard to the potential for positive clinical effects from rAAV1-AAT gene therapy, it must be pointed out that the expression levels observed in this study are more than 200 -fold below the established therapeutic target $(11 \mu \mathrm{M})$ for AAT augmentation therapy. However, the positive safety profile and prolonged duration of transgene expression in this study suggests that this route of delivery remains promising for future product development, including the likely need for a substantial dosage increase. In the highest dosage level cohort, the immune responses detected in these subjects were not linked to clinically significant safety issues. Thus, there does not seem to be any indication for pharmacologic immune suppression or other alteration of the clinical protocols as the clinical trial program for AAV1-AAT continues in the near future.

\section{Methods}

Good Manufacturing Practices Vector Production and Purification. The AAV-AAT expression cassette contains AAV2 inverted terminal repeats on either end of the construct with a $\mathrm{CB}$ enhancer/promoter sequence (containing a cytomegalovirus immediate early enhancer/chicken $\beta$-actin promoter with a hybrid chicken $\beta$-actin/rabbit $\beta$-globin intron) followed by the human AAT CDNA and a bovine growth hormone polyadenylation signal (13). Vector was packaged and purified under current Good Manufacturing Practices using a published cotransfection technique in HEK-293 cells with the pKRAP packaging plasmid, which encodes the other Ad helper genes and the AAV1 rep and cap genes (23). Downstream vector purification was performed using an all-column purification method $(24,25)$.

Clinical Protocol and Informed Consent. Subjects receiving AAT augmentation therapy were asked to discontinue that therapy 28 days (cohort 1) or 56 days (cohorts 2 and 3 ) before vector administration and not resume until at least 11 weeks after vector administration. Subjects returned to the Clinical Research Center on day -1 for repeated studies, and the conformity of each potential 
subject with inclusion and exclusion criteria was confirmed. (For more details refer to $S I$ Text.)

Injection Procedure. The deltoid muscle of the nondominant arm (left arm for right-handed individuals and vice versa) was chosen as the site of injection. Before vector injection, a baseline computed tomography scan of the chest and upper extremity was performed to assess baseline morphology and rule out underlying abnormalities of the upper extremity. Doppler ultrasound was performed over the potential injection site immediately before and during the injection to avoid vascular structures with observable flow. The needle was also directly visualized and the outflow of vector visualized with Doppler ultrasound in real time. The dose was administered in a constant $9.9-\mathrm{mL}$ volume, regardless of vector dose, using lactated Ringers solution to dilute the vector to the proper concentration. At the time of the injection, 9 separate injections of $1.1 \mathrm{~mL}$ each were injected slowly over $1 \mathrm{~min}$ each.

Biodistribution Assays. Blood samples (and semen when available) were obtained at screening and on days 1 (blood only), 3, 14, and 90. DNA was extracted, and vector sequences were detected using Taqman real-time PCR techniques with a previously published primer/probe set directed against a vector-specific CB promoter sequence $(26,27)$. All samples were tested in duplicate, with a third sample containing a positive spike-in control. The technique has a sensitivity of 100 copies per microgram of input DNA.

Measurement of Total and Wild-Type (M-Specific) AAT. Total AAT was measured using a clinically approved nephelometry method, an automated assay in which polyclonal rabbit anti-AAT antibody (Siemens Healthcare Diagnostics) is mixed with serum and the resulting antigen-antibody complexes are detected by light scattering in a nephelometer (Dade-Behring Nephelometer II; Siemens). Mspecific AAT was measured by ELISA, monoclonal antibody was developed in a standard fashion by injecting mice with highly purified PI M1M1 AAT, isolating hybrid clones and identifying clones that made antibody to $M$ but not ZAAT using a dot blot. The M-specific ELISA is a standard double sandwich assay, primary antibody is monoclonal to M AAT, and secondary is rabbit antihuman AAT (Dako). Assays were run on a SPECTRAmax (Molecular Devices) plate reader using a highly purified PI M1M1 AAT (Athens Research). The assay was validated for accuracy, reproducibility, selectivity, lowest limit of quantification, and stability (Fig. S1). Dilutions of serum are added to wells coated with this $\mathrm{mAb}$ at $167 \mathrm{ng} / \mathrm{mL}$ and antigen-antibody complexes detected using a polyclonal rabbit antihuman AAT at $20 \mathrm{ng} / \mathrm{mL}$ followed by a peroxidase-conjugated goat antirabbit lg, with O-phenylenediamine added for colorimetric analysis. The assay is validated for accuracy, repeatability, and precision using a broad range of concentrations and is also validated for serum stability at various temperatures ranging from $24^{\circ} \mathrm{C}$ to

1. Crystal RG (1989) The alpha 1-antitrypsin gene and its deficiency states. Trends Genet 5:411-417.

2. Crystal RG, et al. (1989) The alpha 1-antitrypsin gene and its mutations. Clinical consequences and strategies for therapy. Chest 95:196-208.

3. Ogushi F, et al. (1988) Evaluation of the S-type of alpha-1-antitrypsin as an in vivo and in vitro inhibitor of neutrophil elastase. Am Rev Respir Dis 137:364-370.

4. Brantly ML, et al. (1991) Use of a highly purified alpha 1-antitrypsin standard to establish ranges for the common normal and deficient alpha 1-antitrypsin phenotypes. Chest 100:703-708.

5. Ogushi F, Hubbard RC, Vogelmeier C, Fells GA, Crystal RG (1991) Risk factors for emphysema. Cigarette smoking is associated with a reduction in the association rate constant of lung alpha 1-antitrypsin for neutrophil elastase. J Clin Invest 87:1060-1065.

6. Heresi GA, Stoller JK (2008) Augmentation therapy in alpha-1 antitrypsin deficiency. Expert Opin Biol Ther 8:515-526.

7. Kessler PD, et al. (1996) Gene delivery to skeletal muscle results in sustained expression and systemic delivery of a therapeutic protein. Proc Natl Acad Sci USA 93:14082-14087.

8. Levy MY, Barron LG, Meyer KB, Szoka FC, Jr (1996) Characterization of plasmid DNA transfer into mouse skeletal muscle: Evaluation of uptake mechanism, expression and secretion of gene products into blood. Gene Ther 3:201-211.

9. Clark KR, Sferra TJ, Johnson PR (1997) Recombinant adeno-associated viral vectors mediate long-term transgene expression in muscle. Hum Gene Ther 8:659-669.

10. Song $S$, et al. (1998) Sustained secretion of human alpha-1-antitrypsin from murine muscle transduced with adeno-associated virus vectors. Proc Natl Acad Sci USA 95:14384-14388.

11. Brantly ML, et al. (2006) Phase I trial of intramuscular injection of a recombinant adenoassociated virus serotype 2 alphal-antitrypsin (AAT) vector in AAT-deficient adults. Hum Gene Ther 17:1177-1186.

12. Chao H, Monahan PE, Liu Y, Samulski RJ, Walsh CE (2001) Sustained and complete phenotype correction of hemophilia B mice following intramuscular injection of AAV1 serotype vectors. Mol Ther 4:217-222.

13. Flotte TR, Conlon TJ, Poirier A, Campbell-Thompson M, Byrne BJ (2007) Preclinical characterization of a recombinant adeno-associated virus type 1-pseudotyped vector demonstrates dose-dependent injection site inflammation and dissemination of vector genomes to distant sites. Hum Gene Ther 18:245-256.

14. Manno CS, et al. (2006) Successful transduction of liver in hemophilia by AAV-Factor IX and limitations imposed by the host immune response. Nat Med 12:342-347.

15. Mingozzi F, et al. (2007) Intramuscular administration of an AAV1 vector in humans results in capsid specific T cell responses. Mol Ther 15(Suppl 1):3400 (abstr). $-80^{\circ} \mathrm{C}$. The lower limit of quantification for this assay is $8.8 \mathrm{pM}$, with selectivity and reproducibility at the lower limit of detection $>90 \%$.

Antibody Responses. Serum was obtained at screening and on days 14 and 90 for measurement of neutralizing antibodies to AAV and antibodies to AAT and AAV by ELISA. Neutralizing antibodies were measured as previously described (28). Anti-AAV2 and anti-AAT antibodies were assayed by ELISA as previously described (11). In the case of the anti-AAT antibody assay, the positive control serum was taken from a baboon immunized against human AAT in a prior study (27).

Cellular Immune Responses. Blood was collected and PBMC separated at screening and on days 14 and 90 for measurement of lymphocyte proliferative responses to AAV and AAT antigens as previously described $(26,29)$. For more details refer to SI Text.

In Vitro Stimulation and Staining of Cells and Polychromatic Flow Cytometry. Costimulatory antibodies (anti-CD28 and CD49d, $1 \mu \mathrm{g} / \mathrm{mL}$; BD Biosciences), monensin (Golgistop, $0.7 \mu \mathrm{l} / \mathrm{mL}$; BD Biosciences), brefeldin A (10 $\mu \mathrm{g} / \mathrm{mL}$; SigmaAldrich), and anti-CD107a-PE-Cy5 were added and the cells aliquoted at $1 \mathrm{~mL}$ to a tube containing $2 \mu \mathrm{g}$ of AAV1 peptide libraries. A positive control (Staphylococcus enterotoxin B, $1 \mu \mathrm{g} / \mathrm{mL}$; Sigma-Aldrich) and unstimulated negative control were included in each assay. After fixation, cells were washed twice in perm buffer, stained with antibodies against intracellular markers, and then washed, fixed with PBS containing $1 \%$ paraformaldehyde, and stored at $4{ }^{\circ} \mathrm{C}$ until analysis (performed within $24 \mathrm{~h}$ ). Analysis was performed using FlowJo software (TreeStar) as previously described (31).

Long-Term Follow-Up. Upon completion of this study, each subject will be followed annually for an additional 4 years ( 5 years total) and questioned about the development of any delayed malignant, neurologic, immunologic, hematologic, or other adverse events.

Statistical Analysis. All ELISPOT data were compared using an unequal variance two-tailed Student's $t$ test. Differences were considered statistically significant if $P<0.05$.

ACKNOWLEDGMENTS. We thank Janet King, Glenna Paguio, and Pam Schreck for clinical trial coordination; and the study subjects for their interest and partic ipation in this study. This study was funded by grants from the National Heart Lung, and Blood Institute (R01HL69877 and K24 HL004456-5), the National Institute of Diabetes and Digestive and Kidney Diseases (DK58327 and DK047757 15), to both the University of Florida and the University of Massachusetts and the National Center for Research Resources/National Institutes of Health (U42 RR016586 and MO1 RR000082) to the University of Florida and by support from the study sponsor (Applied Genetic Technologies Corporation) and Alpha 1 Foundation.

16. Calcedo R, Vandenberghe LH, Gao G, Lin J, Wilson JM (2009) Worldwide epidemiology of neutralizing antibodies to adeno-associated viruses. J Infect Dis 199:381-390.

17. Lu $Y$, et al. (2006) Alpha1-antitrypsin gene therapy modulates cellular immunity and efficiently prevents type 1 diabetes in nonobese diabetic mice. Hum Gene Ther 17:625634.

18. Gao G, et al. (2003) Adeno-associated viruses undergo substantial evolution in primates during natural infections. Proc Natl Acad Sci USA 100:6081-6086.

19. Gao GP, et al. (2002) Novel adeno-associated viruses from rhesus monkeys as vectors for human gene therapy. Proc Natl Acad Sci USA 99:11854-11859.

20. Constans J, et al. (1992) Clinical pharmacokinetics of alpha 1-antitrypsin in homozygous PiZ deficient patients. Clin Pharmacokinet 23:161-168

21. Fournel MA, Newgren JO, Betancourt CM, Irwin RG (1988) Preclinical evaluation of alpha-1-proteinase inhibitor. Pharmacokinetics and safety studies. Am J Med 84:43-47.

22. Zamora NP, et al. (2008) Intravenous human plasma-derived augmentation therapy in alpha 1-antitrypsin deficiency: From pharmacokinetic analysis to individualizing therapy. Ann Pharmacother 42:640-646.

23. Grimm D, Kern A, Rittner K, Kleinschmidt JA (1998) Novel tools for production and purification of recombinant adenoassociated virus vectors. Hum Gene Ther 9:2745-2760.

24. Snyder RO, Flotte TR (2002) Production of clinical-grade recombinant adeno-associated virus vectors. Curr Opin Biotechnol 13:418-423.

25. Zolotukhin S, et al. (2002) Production and purification of serotype 1, 2, and 5 recombinant adeno-associated viral vectors. Methods 28:158-167.

26. Poirier A, et al. (2004) Toxicology and biodistribution studies of a recombinant adenoassociated virus 2- $\alpha 1$-antitrypsin vector. Preclinica 2:43-51.

27. Song S, et al. (2002) Intramuscular administration of recombinant adeno-associated virus 2 alpha- 1 antitrypsin (rAAV-SERPINA1) vectors in a nonhuman primate model: Safety and immunologic aspects. Mol Ther 6:329-335.

28. Lin J, Calcedo R, Vandenberghe LH, Figueredo JM, Wilson JM (2008) Impact of preexisting vector immunity on the efficacy of adeno-associated virus-based HIV-1 Gag vaccines. Hum Gene Ther 19:663-669.

29. Hernandez YJ, et al. (1999) Latent adeno-associated virus infection elicits humoral but not cell-mediated immune responses in a nonhuman primate model. J Virol 73:8549-8558.

30. Fu TM, et al. (2007) Evaluation of cellular immune responses in subjects chronically infected with HIV type 1. AIDS Res Hum Retroviruses 23:67-76.

31. Betts MR, et al. (2006) HIV nonprogressors preferentially maintain highly functional HIV-specific CD8+ T cells. Blood 107:4781-4789. 